\title{
GESTÃO DE RESÍDUOS SÓLIDOS: UMA ALTERNATIVA PARA ALIMENTAÇÃO DE ANIMAIS RUMINANTES
}

\author{
Felipe T. C. de Souza1', Maria E. S. de Sousa² Natalia L. B. Santos ${ }^{3}$. \\ 'Docente do Instituto Federal de Alagoas - Campus Penedo, Orientador em Projeto de Extensão do IFAL. e-mail: felipe_thiago1@hotmail.com \\ ${ }^{2}$ Discente do curso técnico integrado em açúcar e álcool - IFAL. Voluntária em Projeto de Extensão do IFAL. e-mail: mariaelayne \\ sousa@hotmail.com \\ ${ }^{3}$ Discente do curso técnico integrado em açúcar e álcool - IFAL. Bolsista em Projeto de Extensão do IFAL. e-mail: nataliabfim@gmail.com
} Autor para correspondência: Felipe Thiago Caldeira de Souza; felipe_thiago1@htmail.com

RESUMO: A Associação de Reabilitação e Equoterapia Santa Clara (ACRESC), localizada na cidade de PenedoAL, possuem 139 pacientes na equoterapia, em sua maioria de baixa renda. Esse método utiliza o cavalo para estimular o desenvolvimento da mente e do corpo para pessoas com disfunção neurológica ou motora. AACRESC possui um alto custo em relação à alimentação dos equinos, em destaque para o fardo de feno, impossibilitando a abertura de novas vagas. Uma alternativa para a diminuição de gastos, que resulte na ampliação de vagas para pessoas de baixa renda, seria a incorporação de resíduos do cotidiano, como o bagaço da cana-de-açúcar, oriundo dos carrinhos de caldo de cana. A aplicação do bagaço na dieta animal proporcionou uma redução dos custos com fardo de feno, melhorou o trato intestinal do animal em comparação com a dieta com $100 \%$ de fardo de feno, além de esmerar o comportamento e diminuir estresse dos equinos contribuindo para aumentar a interação e laços efetivos entre animal e paciente.

PALAVRAS-CHAVE: Resíduos, alimentação animal, ação social.

\section{SOLID WASTE MANAGEMENT: AN ALTERNATIVE TO FEEDING RUMINANT ANIMALS}

ABSTRACT: The Association of Rehabilitation and Equine Therapy Santa Clara (ACRESC), located in the city of Penedo-AL, have 139 patients in equine therapy, mostly low income. This method uses the horse to stimulate the development of the mind and body for people with neurological or motor dysfunction. The ACRESC has a high cost in relation to the feeding of the horses, emphasizing the bale of hay, making it impossible to open new places. An alternative to the reduction of expenses, which results in the increase of vacancies for low-income people, would be the incorporation of everyday residues, such as sugarcane bagasse, from the sugarcane juice carts. The application of bagasse in the animal diet provided a reduction in hay burden costs, improved the intestinal tract of the animal compared to the $100 \%$ bale hay diet, as well as to improve the behavior and decrease stress of the horses contributing to increase the interaction and effective bonds between animal and patient.

KEYWORDS: Waste, animal feed, social action.

\section{INTRODUÇÃO}

A utilização de resíduos da agroindústria, oriundos da lavoura de grãos, fruticultura e de indústrias de biocombustíveis vem sendo utilizados como suplementação alimentar em animais ruminantes. Essa suplementação é estudada sob vários aspectos, como valor nutritivo, digestibilidade, parâmetros ruminais e sanguíneos dos animais, produção e qualidade da carne ou do leite e viabilidade econômica (Oliveira et al., 2012).

O bagaço, resultante da extração do caldo de cana-de-açúcar, é um dos subprodutos mais utilizados como fonte de alimento para os ruminantes, pois além da grande quantidade produzida, sua disponibilidade ocorre exatamente no período de escassez de forragem, principalmente na estiagem do semiárido, onde produtores têm recorrido ao bagaço da cana-deaçúcar distribuído por cooperativas e associações para a complementação da alimentação sendo adicionado junto com a palma forrageira (Leme et al., 2003). Em virtude de suas propriedades, 0 bagaço pode ser incluído em rações para ruminantes, principalmente para corrigir a deficiência em fibra e os distúrbios verificados em animais alimentados com alimentos com baixo teor fibra (Brandão et al., 2003). 
A Associação Comunitária de Reabilitação e Equoterapia Santa Clara (ACRESC), localizada na cidade de Penedo-AL, realiza o tratamento em crianças deficientes por meio da equoterapia, conforme Figura 1. Essa terapia pode ser definida como um método terapêutico e educacional que utiliza o cavalo dentro de uma abordagem interdisciplinar, nas áreas de saúde, educação e equitação, buscando o desenvolvimento biopsicossocial de pessoas portadoras de deficiência e/ou necessidades especiais (SILVA et al., 2014).

Figura 1. Associação Comunitária de Reabilitação e Equoterapia Santa Clara. IFAL, 2016.
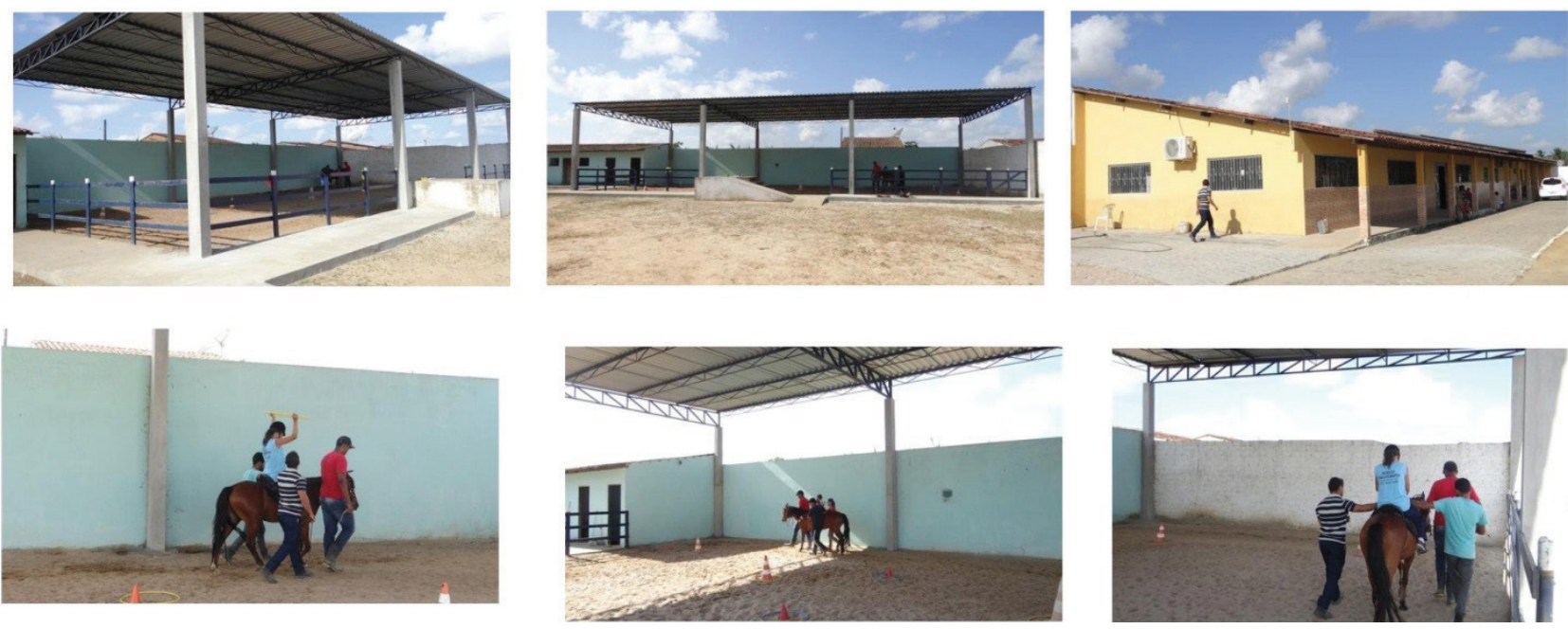

Atualmente, a clínica atende no departamento de equoterapia cerca de 139 pacientes que possuem deficiências e necessidades especiais, sendo atendidos em sua maioria pacientes vinculados ao Sistema Único de Saúde (SUS). A ACRESC apresenta como um dos principais custos financeiros à alimentação dos equinos que compreende o fardo de feno, ração, sal mineral, farelo de trigo e suplemento proteico, minimizando a ampliação de novos atendimentos. Segundo o coordenador de equoterapia, estima-se que aproximadamente $70 \%$ dos custos com a dieta animal é relacionado com o consumo de fardo de feno.

Uma maneira de minimizar os custos com o fardo de feno de forma alternativa e sustentável seria a incorporação de resíduos e subprodutos na alimentação animal, capaz de contribuir para atender as exigências nutricionais, num contexto de viabilidade

econômica, disponibilidade e conservação do meio ambiente (Teixeira et al., 2007).

O caldo de cana-de-açúcar, bebida doce e suave, apreciada por milhares de brasileiros, de norte a sul do país, torna-se uma atividade informal alternativa como fonte de renda, porém atrelada a essa atividade há uma grande geração de resíduos. Esse resíduo é diretamente descartado no lixo sem qualquer tipo de tratamento, promovendo diversos problemas ambientais, tais como, susceptibilidade ao ataque de microrganismos em virtude do teor residual de açúcar, contém grande quantidade de constituintes resistentes à decomposição, tais como celulose $(50 \%)$, hemicelulose $(28 \%)$ e lignina $(10 \%)$, de forma que a sua decomposição em condições naturais seja lenta e aglomeração de bagaço nos centros urbanos (Figura 2).

Figura 2. Resíduo gerado na fabricação de caldo de cana e descarta inadequado do bagaço. IFAL, 2016.
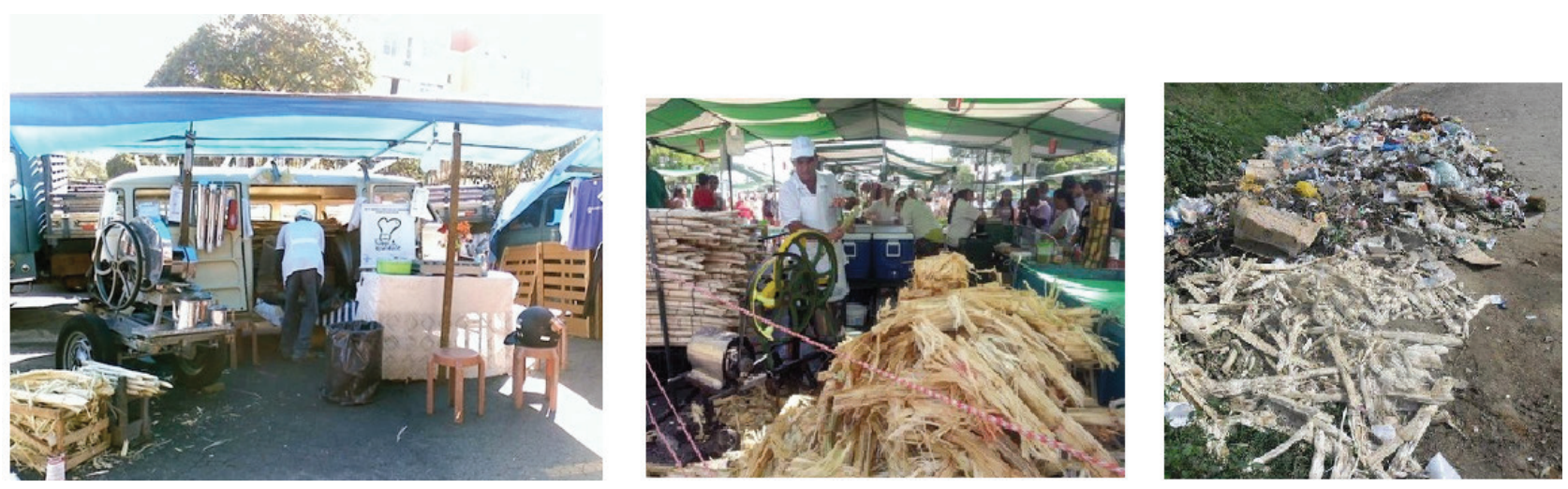
Diante do exposto, foi desenvolvido um projeto de extensão em parceria com a ACRESC com o intuito de reaproveitar o bagaço gerado nos carrinhos de caldo de cana-de-açúcar do município de Penedo. Essa parceria propôs incrementar o bagaço de cana-deaçúcar na dieta dos equinos da clínica de reabilitação e equoterapia, visando a redução dos gastos com fardo de feno e minimizar os impactos ambientais causados por esse resíduo, com consequente aumento de vagas para atendimentos a pessoas de baixa renda.

\section{MATERIAL E MÉTODOS}

Inicialmente a matéria-prima foi coletada nos estabelecimentos onde comercializam caldo de cana na cidade de Penedo - AL. Após a coleta, o resíduo foi exposto ao sol, por aproximadamente 5 horas, para diminuir sua umidade e consequentemente retardar a degradação bioquímica do bagaço por microrganismos indesejáveis. Em seguida, 0 bagaço seco passou pelo processo de trituração na forrageira, com o intuito de melhorar a digestibilidade dos equinos. Por fim, o bagaço triturado foi ensacado e enviado para ACRESC, para ser incrementado na dieta dos equinos durante três dias na semana, alimentando-os duas vezes ao dia, sem que houvesse mudança significativa na dieta dos equinos, apenas suplementação com sal mineral.

\section{RESULTADOS E DISCUSSÃO}

A inclusão do bagaço na dieta animal, mesmo que de maneira parcial, implicou numa redução de $30 \%$ do gasto relacionado ao fardo do feno como descrito na Tabela 1. Todavia, a substituição integral do fardo de feno não é possível, haja vista que o bagaço de canade-açúcar apresenta um valor nutritivo inferior quando comparado ao fardo de feno (Burgi, 1995).

Tabela 1. Componentes da Dieta dos Equinos e Seus Respectivos Valores

\begin{tabular}{|c|c|c|c|c|}
\hline Componentes da dieta & $\begin{array}{c}\text { Quantidade } \\
\text { anterior } \\
\text { (Mensal) }\end{array}$ & $\begin{array}{l}\text { Quantidade } \\
\text { posterior } \\
\text { (Mensal) }\end{array}$ & $\begin{array}{l}\text { Valor Anterior } \\
\text { (Mensal) }\end{array}$ & $\begin{array}{l}\text { Valor Posterior } \\
\text { (Mensal) }\end{array}$ \\
\hline Farelo de Trigo & $80 \mathrm{Kg}$ & $80 \mathrm{Kg}$ & $\mathrm{R} \$ 56,00$ & $R \$ 56,00$ \\
\hline Sal Mineral & $50 \mathrm{Kg}$ & $50 \mathrm{Kg}$ & $R \$ 50,00$ & $R \$ 50,00$ \\
\hline Ração & $800 \mathrm{Kg}$ & $800 \mathrm{Kg}$ & $\mathrm{R} \$ 1.200,00$ & $\mathrm{R} \$ 1.200,00$ \\
\hline Fardo Feno & $1.460,00 \mathrm{Kg}$ & $1.024,00 \mathrm{Kg}$ & $\mathrm{R} \$ 1500,00$ & $\mathrm{R} \$ 1.000 .00$ \\
\hline Bagaço da Cana-de-Açúcar & 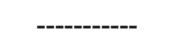 & $144 \mathrm{Kg}$ & 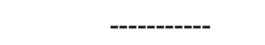 & 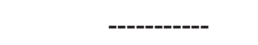 \\
\hline Valor Total & & & $\mathrm{R} \$ 2.816,00$ & $\mathrm{R} \$ 2.306,00$ \\
\hline
\end{tabular}

De maneira geral, a sua incorporação do bagaço na alimentação dos equinos favorece o intestino do animal deixando-o mais "leve" devido à alta taxa de fibrose deste resíduo. Desse modo, foi observado que após adição do bagaço os equinos apresentaram melhor disponibilidade tornando-os mais dóceis e agradáveis com os pacientes, além da melhora na pelagem do animal. Essa característica está associada a uma dieta rica em fibra, de modo que o animal fica mais relaxado, fica menos aborrecido e o seu comportamento é muito mais tranquilo, evitando as chamadas estereotipias, estado em que 0 animal come materiais estranhos como areia ou madeira, fazendo movimentos anormais como a "birra de urso" e comportamento mais nervoso. Além disso, a utilização do bagaço, insumo rico em açúcares, proporciona energia necessária para os equinos realizem suas atividades e funções fisiológicas (Bonsfills, 2013).

No contexto ambiental, o reaproveitamento de um resíduo descartado de maneira aleatória, sem qualquer tratamento, poderia causar impactos negativos ao meio ambiente, desta forma o incremento do bagaço na dieta animal minimiza problemas de contaminação, principalmente do solo. A diminuição de gastos com o fardo de feno promoveu a redução de custos, de maneira que, a clínica poderá vislumbrar a oferta de novas vagas para pessoas de baixa renda, contribuído para inclusão social de pessoas com deficiências e necessidades especiais.

A substituição do bagaço, mesmo que de forma parcial, demonstrou resultados interessantes no ponto de vista ambiental, minimizando o descarte inadequado deste resíduo, econômico com a redução nos custos com fardo de feno e social através da inclusão de novas vagas para pessoas carentes na clinica de reabilitação e equoterapia, estando em conformidade com as políticas de extensão do Instituto Federal de Alagoas. 


\section{AGRADECIMENTOS}

APró-Reitoria de Extensão pela disponibilidade das bolsas de estudo. A Associação Comunitária de Reabilitação e Equoterapia Santa Clara (ACRESC) e ao Coordenador de Equoterapia e Equitação Rodrigo Regueira.

\section{REFERÊNCIAS BIBILOGRÁFICAS}

OLIVEIRA J.N. Compostagem e Vermicompostagem de bagaço de cana-de-açúcar da produção de cachaça de alambique de Salinas-MG. Dissertação de mestrado, Programa de Pós-Graduação em Desenvolvimento Regional e Meio Ambiente, Dissertação de mestrado, 2010.

PRUDENTE, D.S.; MASUNAGA, E.; MORAES, P.H.G.; OLIVEIRA, R. Utilização do bagaço da cana-de-açúcar na composição da dieta no confinamento bovino. Revista Unilins, 2010, 1, 1-7.

SILVA, A.C.; SOUSA, C.S. A utilização da equoterapia no tratamento da síndrome de down: uma revisão sistemática. Getec, 2014, 3, 68-77.
SOARES, M.S.; PIRES, A.J.V.; SILVA, L.G.; GUIMARÃES, J.O.; MACHADO, T.C.; FRAZÃO, O.S. Utilização do bagaço de cana-de-açúcar na alimentação de ruminantes. Revista Eletrônica Nutritime, 2015, 12, $3837-3855$.

TEXEIRA, F.A.; PIRES, A.V.; NASCIMENTO, P.V.N. Bagaço de cana-de-açúcar na alimentação de bovinos (Sugarcane pulp in the feeding of bovine). Revista Electrónica de Veterinária, 2007, 6, 1695-7504.

FERREIRA, W.M. Os componentes da parede celular vegetal na nutrição. In: Reunião anual da Sociedade Brasileira de Zootecnia, 31. Simpósio Internacional de Produção de ruminates. Maringá, 1994. Anais... p: 85113.

BURGI, R. Utilização de resíduos culturais e de beneficiamento de na alimentação de bovinos. Simpósio sobre nutrição de bovinos da FEALQ $\left(6^{\circ}\right.$.), Anais..., 1995. PiracicabaSP, p. 153-169. 


\title{
RELATO DE EXPERIÊNCIA: O CASO DA PRODUÇÃO DE COCO ORGÂNICO DA FAZENDA NOSSA SENHORA APARECIDA EM PARAIPABA, CEARÁ
}

\author{
Letícia Kenia Bessa de Oliveira ${ }^{1}$, Rafael Santiago da Costa ${ }^{1}$, Daniela Queiroz Zuliani², Leidiane Marques Maciel', \\ Francisca Evelice Cardoso de Souza ${ }^{1}$, Ana Isabel Pinheiro ${ }^{3}$, Aiala Vieira Amorim² \\ 'Estudante de Graduação em Agronomia, Instituto de Desenvolvimento Rural (IDR), Universidade da Integração Internacional da \\ Lusofonia Afro-Brasileira (UNILAB), Av. Abolição, Redenção-CE. CEP: 62.790-000 \\ 2Professora, Instituto de Desenvolvimento Rural (IDR), UNILAB, Av. Abolição, Redenção-CE. CEP: 62.790-000 \\ ${ }^{3}$ Mestranda em Sociobiodiversidade e Tecnologias Sustentáveis, UNILAB, Av. Abolição, Redenção-CE. CEP: 62.790-000
}

*Autor para correspondência: Aiala Vieira Amorim; aialaamorim@unilab.edu.br

\begin{abstract}
RESUMO: A Agroecologia apresenta princípios que permitem o estudo, a análise, o desenho e o manejo de agroecossistemas, com 0 intuito de promover agricultura mais sustentável. Objetivou-se, com 0 presente trabalho, socializar uma experiência adquirida por alunos do curso de Agronomia da Universidade da Integração Internacional da Lusofonia Afro-Brasileira (Unilab), onde se buscou conhecer como era realizado o cultivo de coco orgânico e correlacionar as atividades desempenhadas com os conceitos e os princípios defendidos pela Agroecologia. As informações foram obtidas através de uma caminhada transversal realizada na área de cultivo da fazenda Nossa Senhora Aparecida, Paraipaba, Ceará. Esta permitiu o conhecimento de técnicas e manejos para produção agroecológica, bem como formas de combate às pragas através de métodos menos danosos ao meio ambiente. Percebeu-se a sinergia entre os fatores determinantes, recursos e processos que compõem a propriedade e a importância dessa interação para maximizar a produção agrícola. Apesar destes pontos positivos, a propriedade apresenta um expressivo ponto negativo, a prática da monocultura, fazendo-se necessário 0 aumento da biodiversidade.
\end{abstract}

PALAVRAS-CHAVE: Agroecologia, meio ambiente, biodiversidade, monocultura.

\section{EXPERIENCE REPORT: THE CASE OF ORGANIC COCONUT PRODUCTION OF THE FARM NOSSA SENHORA APARECIDA IN PARAIPABA, CEARÁ}

\begin{abstract}
Agroecology presents principles that allow the study, analysis, design and management of agroecosystems, with the aim of promoting more sustainable agriculture. The objective of this work was to socialize an experience acquired by students of the Agronomy course of the University of the Integration International of Lusophony Afro-Brazilian (Unilab), where sought to know how the planting of the organic coconut was carried out and to correlate the performed activities With the concepts and principles defended by Agroecology. The information was obtained through a transversal walk carried out in the area of cultivation of Nossa Senhora Aparecida farm, Paraipaba, Ceará. This allowed the knowledge of techniques and management for agroecological production, as well as ways of combating pests through methods less harmful to the environment. The synergy between the determinants, resources and processes that make up the property and the importance of this interaction to maximize agricultural production was perceived. Despite these positive points, the property presents an expressive negative point, the practice of monoculture, making it necessary to increase biodiversity.
\end{abstract}

KEYWORDS: Agroecology, environment, biodiversity, monoculture.

\section{INTRODUÇÃO}

O modelo agrícola convencional, centrado no uso abusivo de recursos naturais e de agroquímicos de síntese, permitiu aumentar a produção e a produtividade de alguns cultivos em determinadas regiões, porém vem causando fortes agressões e danos irreparáveis ao meio ambiente (Teixeira, 2005). Para Miller (2008), o pacote tecnológico (uso de máquinas e insumos industriais, e outras técnicas, a fim de viabilizar a produção extensiva) introduzido a 